Article

\title{
Improved Method for Distributed Parameter Model of Solenoid Valve Based on Kriging Basis Function Predictive Identification Program
}

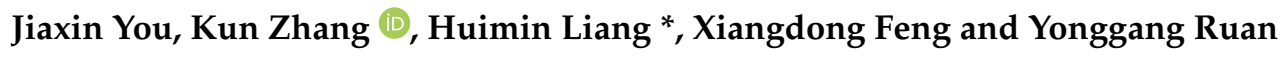 \\ School of Electrical Engineering and Automation, Harbin Institute of Technology, Harbin 150001, China; \\ ibic2013@126.com (J.Y.); zhangkunhit2020@163.com (K.Z.); 20S106170@stu.hit.edu.cn (X.F.); \\ ruanyonggang6@163.com (Y.R.) \\ * Correspondence: hitra@hit.edu.cn
}

Citation: You, J.; Zhang, K.; Liang, H.; Feng, X.; Ruan, Y. Improved Method for Distributed Parameter Model of Solenoid Valve Based on Kriging Basis Function Predictive Identification Program. Actuators 2021, 10, 10. https://doi.org/ $10.3390 /$ act 10010010

Received: 25 November 2020

Accepted: 4 January 2021

Published: 7 January 2021

Publisher's Note: MDPI stays neutral with regard to jurisdictional clai$\mathrm{ms}$ in published maps and institutional affiliations.

Copyright: $\odot 2021$ by the authors. Licensee MDPI, Basel, Switzerland. This article is an open access article distributed under the terms and conditions of the Creative Commons Attribution (CC BY) license (https:// creativecommons.org/licenses/by/ $4.0 /)$.

\begin{abstract}
In this paper, a method for the improvement of the calculation accuracy of the distributed parameter model (DPM) of electromagnetic devices is proposed based on the kriging basis function predictive identification program (PIP). Kriging is mainly an optimal interpolation method which uses spatial self-covariance, and takes a polynomial as the basis function. The accuracy of the krigingbased surrogate model can be improved by adjusting the related functions and hyperparameters. Based on the DPM of a solenoid valve, there exist certain errors in the estimation. They can be summarized as follows: Firstly, the estimation error of magnetic flux leakage (MFL) permeance is caused directly by the deviation of the magnetic flux tube due to the segmented magnetic field line. Secondly, the estimation error of soft magnetic resistance because of the nonlinearity of the permeability of soft magnetic material leads to the change of soft magnetic resistance alongside the magnetic flux. In this paper, an improved kriging error correction method is applied to modify the leak permeance and soft magnetic resistance calculation. The kriging basis function is adjusted to adapt to the data curve of the MFL permeance error data. The calculated MFL permeance data are compared with the error variation data to select the appropriate basis function. To improve the computational efficiency, the PIP is proposed to select the appropriate basis function. The modified MFL permeance data and soft magnetic resistance are substituted into the DPM for improving the computational accuracy and efficiency of the solenoid valve.
\end{abstract}

Keywords: kriging; distributed parameter model; magnetic flux leakage; permeance; predictive identification program

\section{Introduction}

The optimization design of modern solenoid valves faces more and more challenges with increasing performance requirements to realize higher latching force, higher output force, and more extensive robustness [1-9]. As a means of simulation, the FEM method has the absolute advantage of accuracy, and the disadvantage of efficiency. The distributed parameter model (DPM) method can effectively speed up the calculation, and kriging models can decrease error. The DPM and kriging models have become important factors affecting the design efficiency and accuracy of solenoid valves. Therefore, evaluating the applicability of the kriging model has an important theoretical significance and a practical engineering value for product design optimization [10-15].

With the increasing levels of computational technology and theoretical analysis for the DPM, the kriging model, whose inputs affect its outputs, plays an important role in engineering design and error correction [16-21]. The calculation accuracy of air leakage permeance and the soft magnetic resistance has an effect on the electromagnetic force calculation accuracy of solenoid valves. To reduce the relative error and improve the accuracy of target prediction based on sample data, various data fitting methods based 
on the kriging model have been proposed. In the design and optimization of products, kriging has a good performance in error correction [22-28]. An adaptive finite difference weighted essentially non-oscillatory (WENO) method with Gauss-kriging reconstruction is proposed to reduce dissipation in smooth regions of flow, while preserving high resolution around discontinuities for hyperbolic systems of conservation laws [29]. The work in [30] proposed a kriging model-based method for the reliability design and optimization of planetary gear, using a genetic algorithm. The kriging model was used to establish the gear reliability model to simplify the reliability calculation in this method [30]. Shin et al. performed an optimization study of response time to improve the dynamic performance of a direct-acting solenoid valve based on a kriging model [31]. A kriging metamodel-based multi-objective optimization strategy has been employed to optimize the valve-plate shape of the axial piston pump [32]. The kriging method was also used to build a surrogate model, which presents the relationship between dynamic responses and dynamic simulation of the valve train [33]. Many of the works where the kriging model has been adopted for error calculation mainly focus on the calculation errors associated with replacing the original analytical model with the kriging-based surrogate model.

In this paper, a method for the improvement of the calculation efficiency and accuracy of the DPM of electromagnetic devices is proposed based on the kriging basis function predictive identification program (PIP). To obtain an improved DPM performance, an appropriate basis function is selected by contrasting various basis functions with error item curves of air leakage permeance. To further improve the calculation efficiency, the PIP is introduced to prejudge the error item curves against standard functions. The modified leakage permeance and the soft magnetic resistance data are considered in the DPM of the electromagnetic device to calculate the electromagnetic force, thereby verifying the method proposed in this paper.

\section{The Error Correction Method of DPM Based on Kriging Model}

The kriging model is a global surrogate model derived from geostatistics, and it can mathematically provide an optimal linear unbiased estimation. This makes it an appropriate method to achieve the primary goal of this paper. To estimate the linear, unbiased and minimum estimated variance using kriging, the geometric features of information sample shape, size and space on samples are taken into account. Nonetheless, kriging is still a smooth interpolation method [34,35]. The electromagnetic calculation software is Matlab in this paper.

The kriging model can be summarized in two parts, which are represented by

$$
y(x)=\sum_{j=1}^{p} \beta_{j} f_{j}(x)+Z(x)
$$

where $f_{j}(x)$ is the basis, $\beta_{j}$ is the regression coefficient, $Z(x)$ is the stochastic distribution function.

A solenoid valve with a fan-shaped permanent magnet (PM) is selected as the example in this study, and its electromagnetic system is shown in Figure 1. The armature is a moving part, the angle of the fan-shaped PM is $20^{\circ}$, its inner radius is $8.5 \mathrm{~mm}$, its outer radius is $17.5 \mathrm{~mm}$ and its height is $8 \mathrm{~mm}$. The solenoid valve rated coil ampere-turns equal 1800 AT. The PM is divided into seven segments. The magnetic flux leakage (MFL) permeances of the flux tube from the outer radius side to the inner radius side are $G_{1}, G_{2}, G_{3}, G_{4}, G_{5}, G_{6}$ and $G_{7}[36-40]$.

The solenoid valve is excited in $0 \mathrm{AT}$, and its finite element method (FEM) responses are compared with the unmodified DPM, as shown in Figure 2. There are 20 sample points for every curve. 


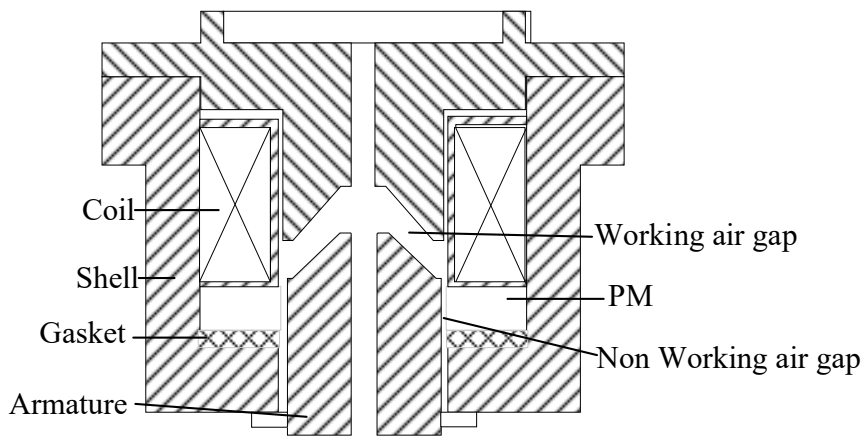

(a)

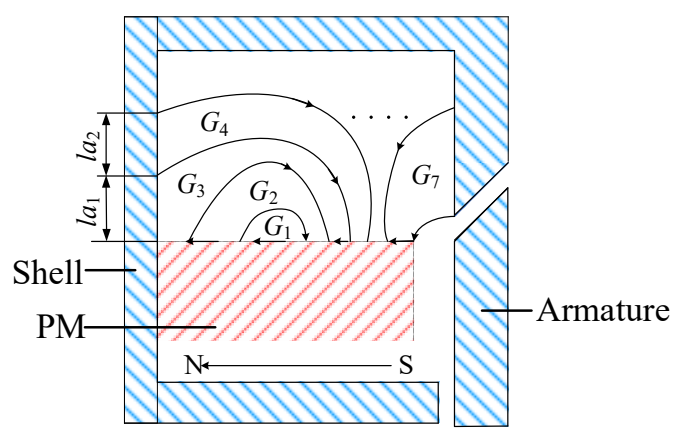

(b)

Figure 1. Solenoid valve with fan-shaped permanent magnet (PM): (a) sectional view of solenoid valve; (b) magnetic flux tube diagram (half of Figure 1a).

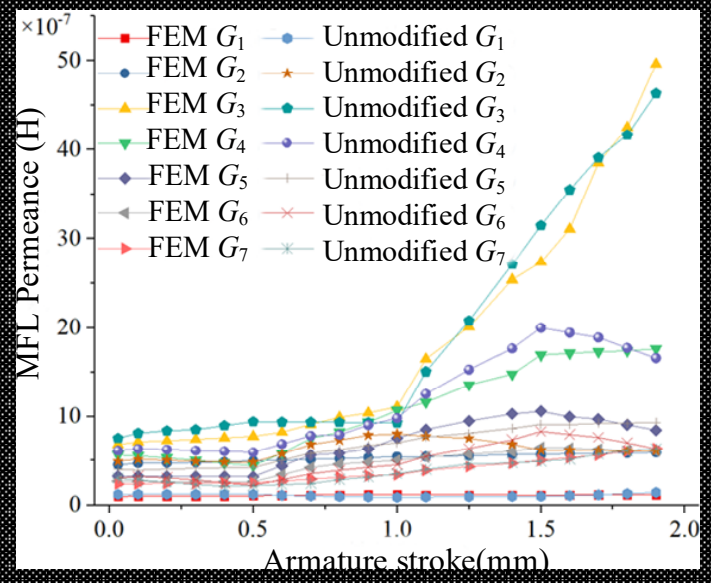

Figure 2. The leakage permeance of FEM and before error modifying the distributed parameter model.

The FEM and DPM data are subtracted to obtain the MFL permeance error terms (i.e., $\Delta G_{1}, \Delta G_{2}, \Delta G_{3}, \Delta G_{4}, \Delta G_{5}, \Delta G_{6}$ and $\Delta G_{7}$ ), which are then stored in the error matrix $E r$. The MFL permeance data are shown in Figure 3 which reveal a non-monotonic variation.

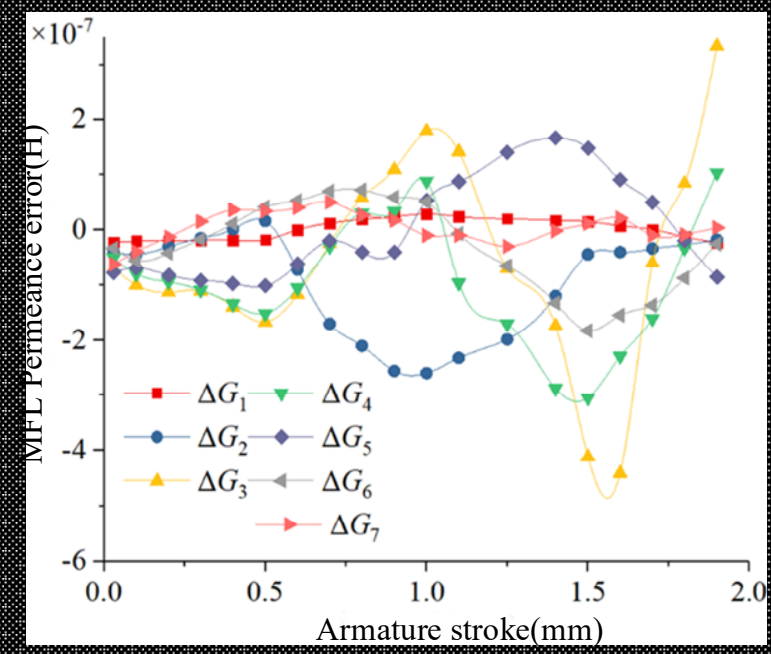

Figure 3. The leakage permeance error values in 0 AT.

To further improve the error correction efficiency, multi-basis functions are applied to the adaptive error curve. The kriging basis functions are represented as follows: 


\section{Fourier Function}

$$
f(x)=\sum_{i}^{n}\left[a_{i} \sin \left(d_{i} w i\right)+b_{i} \sin \left(d_{i} w i\right)\right] .
$$

Polynomial Function

$$
f(x)=1+\sum_{i=1}^{n} d_{i}+\sum_{i=1}^{n} d_{i}^{2}+\sum \sum_{i<j} d_{i} d_{j} .
$$

Exponential Function

$$
f(x)=e^{\sum_{i=1}^{n}\left(-\theta_{i},\left|d_{j}\right|\right)}
$$

Gaussian Function

$$
f(x)=e^{\sum_{i=1}^{n}\left(-\theta_{i},\left|d_{j}\right|^{2}\right)},
$$

where $\left|d_{\mathrm{i}}\right|$ is the distance between the known and predicted quantities.

The basis function output characteristics can be determined using the key nodes and the output of the model. Given a function $k\left(x_{1}, x_{2} \ldots x_{n}\right)$ and an input parameter $x_{1}, x_{2} \ldots$ $x_{n}$, the domain of the function can be expressed as follows:

$$
\left\{\begin{array}{l}
X_{1} \in\left[x_{1}^{0}-\Delta x_{1}, x_{1}^{0}+\Delta x_{1}\right] \\
X_{2} \in\left[x_{2}^{0}-\Delta x_{2}, x_{2}^{0}+\Delta x_{2}\right] \\
\vdots \\
X_{n} \in\left[x_{n}^{0}-\Delta x_{n}, x_{n}^{0}+\Delta x_{n}\right]
\end{array},\right.
$$

where $x_{1}, x_{2} \ldots x_{n}$ are the coordinate positions of magnetic lines of flux with armature displacement, $x_{10}, x_{20} \ldots x_{n 0}$ are the center of fluctuations, $\Delta x_{1}, \Delta x_{2} \ldots \Delta x_{n}$ are the ranges of fluctuations. The basis function can be simplified to:

$$
\Delta F \approx \sum_{i=1}^{n} \Delta F_{i}+\Delta^{2}(\Delta x),
$$

where $\Delta F$ is the function output, $\Delta^{2}(\Delta x)$ is the higher derivative of the input parameter fluctuations. Given that a function relation exists between $k\left(x_{1}, x_{2} \ldots x_{n}\right)$ and $\Delta x_{1}, \Delta x_{2} \ldots$ $\Delta x_{n}, k\left(x_{1}, x_{2} \ldots x_{n}\right)$, this function relation can be described as follows:

$$
k\left(x_{1}^{0}, x_{2}^{0} \ldots x_{n}^{0}\right)=\left.\sum_{x_{1}^{0}-\Delta x_{1}, x_{2}^{0}-\Delta x_{2} \ldots x_{n}^{0}-\Delta x_{n}}^{x_{1}^{0}+\Delta x_{1}, x_{2}^{0}+\Delta x_{2} \ldots x_{n}^{0}+\Delta x_{n}} k\left(x_{1}, x_{2} \ldots x_{n}\right) w\right|_{x_{1}, x_{2} \ldots x_{n}}
$$

where $w$ is the weight coefficient. For every node in the count, $w=1$. However, if the sample points are not key nodes, then $0<w<1$. Given that, the boundary condition of the input parameter is as follows:

$$
\left\{\begin{array}{l}
x_{1 l} \leq x_{1} \leq x_{1 h} \\
x_{2 l} \leq x_{2} \leq x_{2 h} \\
\vdots \\
x_{n l} \leq x_{n} \leq x_{n h}
\end{array},\right.
$$

where $x_{i l}, x_{i h}$ are the top and bottom limitations of the $i$ th input value. Combining Equations (7)-(9), $\Delta F_{\mathrm{i}}$ can be defined as:

$$
\Delta F_{i}=\left.\left.\sum_{\left(x_{l h}, x_{l h} \ldots x_{l h}\right)}^{\left(x_{1 h}, x_{2 h} \ldots x_{n h}\right)} w \Delta F\left(\Delta x_{i}\right)\right|_{x_{1}, x_{2} \ldots x_{n}} G\left(x_{1}, x_{2} \ldots x_{n}\right)\right|_{x_{1}^{0}, x_{2}^{0} \ldots x_{n}^{0}},
$$


where $G\left(x_{1}, x_{2} \ldots x_{n}\right)$ is the interpolating function of $x_{1}, x_{2} \ldots x_{n}$. Substituting $G\left(x_{1}, x_{2} \ldots\right.$ $\left.x_{n}\right)$ into Equations (7) and (8), the following equations are obtained:

$$
\begin{aligned}
& \left.G\left(x_{1}, x_{2} \ldots x_{n}\right)\right|_{\left(x_{1}^{0}, x_{2}^{0} \ldots x_{n}^{0}\right)}=e^{\sum_{i=1}^{n}\left(-\theta_{i},\left|d_{i}\right|\right),} \\
& \left.G\left(x_{1}, x_{2} \ldots x_{n}\right)\right|_{\left(x_{1}^{0}, x_{2}^{0} \ldots x_{n}^{0}\right)}=e^{\sum_{i=1}^{n}\left(-\theta_{i},\left|d_{i}\right|^{2}\right),}
\end{aligned}
$$

where $d_{i}=\left|x-x_{i}\right|, x$ is the dependent variable, $\theta_{i}$ is the PM segment positions with respect to the armature displacement. The armature stroke is $2 \mathrm{~mm}$, and $\theta_{i}$ can be obtained by particle swarm optimization (PSO) based on 20 nodes.

Based on the MFL permeance error data, and adopting a periodic function (i.e., Fourier series) for $\Delta G_{3}$ fitting, it can be written as follows:

$$
f(x)=a_{0}+\sum_{i=1}^{4}\left[a_{i} \cos (i x w)+b_{i} \sin (i x w)\right],
$$

where $x$ is the armature stroke, $f(x)$ is the dependent variable of the MFL permeance. $a_{0}=-857, a_{1}=1384, b_{1}=-45.96, a_{2}=-71.14, b_{2}=43.84, a_{3}=212.3, b_{3}=-17.28, a_{4}=-28.1$, $b_{4}=2.529, w=0.1202$. Taking the derivative of Equation (13), $\mathrm{d} f(x) / \mathrm{d} x$ has four solutions defined in the range 0 to 2 .

By adopting a polynomial function for $\Delta G_{3}$ fitting, it can be written as follows:

$$
f(x)=p_{1} x^{7}+p_{2} x^{6}+p_{3} x^{5}+p_{4} x^{4}+p_{5} x^{3}+p_{6} x^{2}+p_{7} x+p_{8}
$$

where $p_{1}=-5.34 \times 10^{-6}, p_{2}=3.26 \times 10^{-5}, p_{3}=-7.49 \times 10^{-5}, p_{4}=8.1 \times 10^{-5}, p_{5}=-4.26$ $\times 10^{-5}, p_{6}=1.08 \times 10^{-6}, p_{7}=-1.33 \times 10^{-6}, p_{8}=-3.92 \times 10^{-8}$. Taking the derivative of Equation (14), i.e., $\mathrm{d} f(x) / \mathrm{d} x$, yields four solutions.

By adopting an exponential function for $\Delta G_{3}$ fitting, it can be written as follows:

$$
f(x)=a e^{b x}+c e^{d x}
$$

where $a=-7.591 \times 10^{-7}, b=4.862, c=7.583 \times 10^{-7}, d=4.863$. Taking the derivative of Equation (15), i.e., $\mathrm{d} f(x) / \mathrm{d} x$, has only one solution. It is obvious that the exponent is unfit for $\Delta G_{3}$ fitting.

By adopting a Gaussian function for $\Delta G_{3}$ fitting, it can be written as follows:

$$
f(x)=a_{1} e^{-\frac{\left(x-b_{1}\right)^{2}}{2 c_{1}^{2}}}+a_{2} e^{-\frac{\left(x-b_{2}\right)^{2}}{2 c_{2}^{2}}}+a_{3} e^{-\frac{\left(x-b_{3}\right)^{2}}{2 c_{3}^{2}}}+a_{4} e^{-\frac{\left(x-b_{4}\right)^{2}}{2 c_{4}^{2}}}+a_{5} e^{-\frac{\left(x-b_{5}\right)^{2}}{2 c_{5}^{2}}},
$$

where $a_{1}=1.28 \times 10^{-6}, b_{1}=1.906, c_{1}=0.2655, a_{2}=0, b_{2}=10.97, c_{2}=0.03011, a_{3}=2.89 \times$ $10^{-5}, b_{3}=1.29, c_{3}=0.6738, a_{4}=-2.87 \times 10^{-5}, b_{4}=1.302, c_{4}=0.6887, a_{5}=-0.6322 \times 10^{-7}$, $b_{5}=0.5452, c_{5}=0.1176$. Taking the derivative of Equation (16), i.e., $\mathrm{d} f(x) / \mathrm{d} x$, yields four solutions.

Figure 4 shows the contrasting condition between basis functions and MFL permeance error $\Delta G_{3}$. It is obvious that there is a large error between the exponential function-based fitted curve and $\Delta G_{3}$. The mean error for this particular case is over $100 \%$. The polynomial function-based fitted curve, Fourier function-based fitted curve and Gaussian functionbased fitted curve have mean errors reaching $38.4 \%, 30.1 \%$ and $14.2 \%$, respectively. 


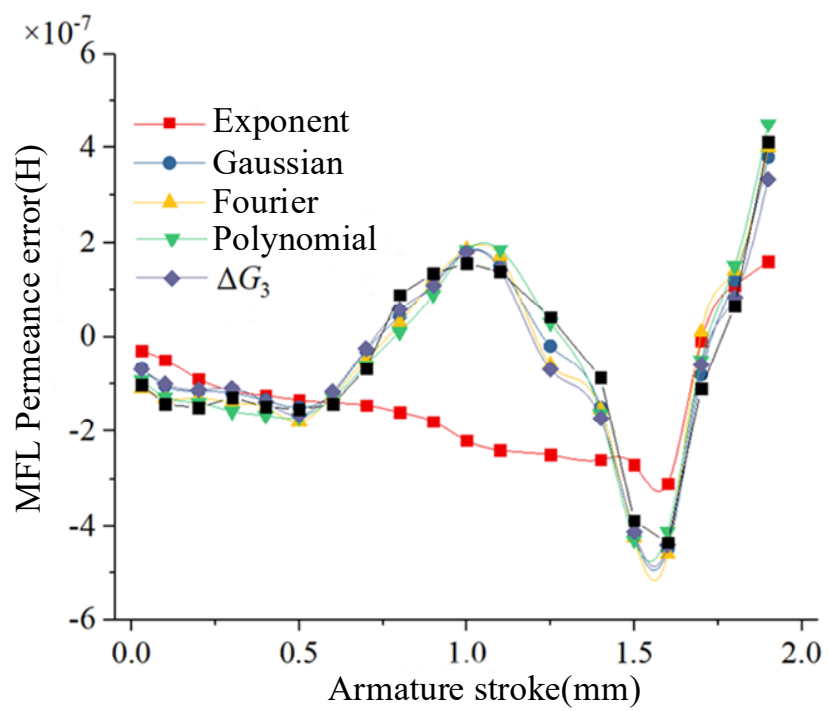

Figure 4. The comparison of $\Delta \mathrm{G} 3$ calculations and FEM data on basis functions.

To further improve computational efficiency, the reliability index of samples is calculated by four algorithms to achieve optimal basis function. The algorithms are:

1. Particle Swarm Optimization(PSO)

2. Response Surface (RS)

3. Linear Second order moment (LS)

4. Monte Carlo (MC)

The relative error of the basis functions can be obtained by taking $\mathrm{MC}$ as the base. Firstly, the failure probability of the samples is calculated, then the reliability index can be obtained using the calculation results. The reliability index is calculated by the MC method and it is used as the standard with a termination condition of $10^{-7}$.

Table 1 shows the reliability index of $\Delta G_{3}$.

Table 1. The basis function model reliability index of $\Delta \mathrm{G} 3$.

\begin{tabular}{ccccc}
\hline Function & Algorithm & Iteration & Count & Time (s) \\
\hline \multirow{5}{*}{ Gaussian } & PSO & 132 & 660 & 987 \\
& RS & 189 & 945 & 1229 \\
& LS & 147 & 588 & 1143 \\
& MC & - & $10^{5}$ & 2961 \\
Fourier & PSO & 162 & 810 & 1328 \\
& RS & 198 & 990 & 1843 \\
& LS & 204 & 816 & 1687 \\
\multirow{5}{*}{ Polynomial } & MC & - & $10^{5}$ & 3063 \\
& PSO & 153 & 765 & 1125 \\
& RS & 207 & 1035 & 2063 \\
& LS & 192 & 768 & 1763 \\
& MC & - & $10^{5}$ & 3012 \\
\hline
\end{tabular}

Table 1 shows that Gaussian function with PSO has the smallest count for $\Delta G_{3}$, while the Fourier function with PSO has the smallest count for $\Delta G_{1}$.

$\Delta G_{3}$ is presented as an example. The polynomial function is adopted for the armature stroke, Gaussian error data and $\Delta G_{3}$. This function can be written as follows:

$$
\Delta G_{3}=p_{00}+p_{10} x+p_{01} y^{3}+p_{20} x+p_{11} x y,
$$


where $p_{00}=-2.057 \times 10^{-7}, p_{10}=7.069 \times 10^{-7}, p_{01}=-0.02261, p_{20}=-4.141 \times 10^{-7}$, $p_{11}=0.3128 . x$ is the armature stroke, $y$ is the Gaussian error data. Figure 5 shows Gaussian error data fitting.

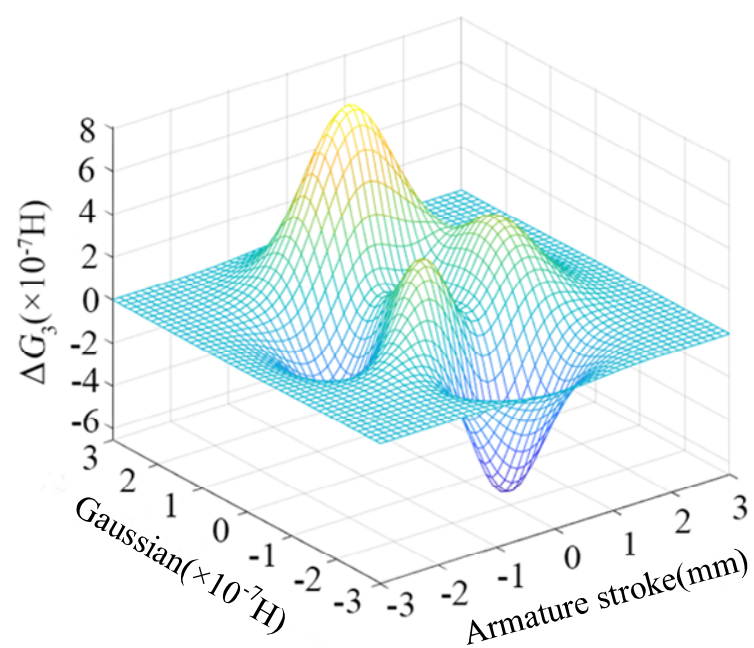

Figure 5. The basis function of Gaussian error data fitting.

The MFL permeance of the solenoid valve $G_{3}$ can be improved. Now, assuming that $P$ is the FEM data matrix and $Q$ is the DPM data matrix, Er can be written as follows:

$$
E r=P-Q
$$

If $\Delta G$ depicts the permeance error of the sampling point, $E r$ can be derived as follows:

$$
E r=\left[\begin{array}{cccc}
\Delta G_{11} & \Delta G_{12} & \cdots & \Delta G_{1 m} \\
\Delta G_{21} & \ddots & & \Delta G_{2 m} \\
\vdots & & \ddots & \vdots \\
\Delta G_{n 1} & \Delta G_{n 2} & \cdots & \Delta G_{n m}
\end{array}\right]
$$

where $n$ is the PM segment number, $m$ is the sampling point position. $i=1,2, \ldots n . j=1,2$, ... $m$.

The basis function can be defined as $f_{k}\left(x_{1}, x_{2} \ldots x_{q}\right), k=1,2,3, \ldots n$, where $q$ is the dimension. $f_{k}\left(x_{1}, x_{2} \ldots x_{q}\right)$. The function can be written as:

$$
f\left(x_{1}, x_{2}, \ldots, x_{q}\right)=\min \left\{f_{k}\left(x_{1}, x_{2}, \ldots, x_{q}\right)-\operatorname{Er}[k ;]\right\} .
$$

Based on Equation (20), the polynomial function is adopted for the armature stroke, $l$, $\Delta G$ and $f\left(x_{1}, x_{2} \ldots x_{q}\right)$. This function is written as follows:

$$
\Delta G=p_{00}+p_{10} l+p_{01} f^{3}\left(x_{1}, x_{2} \ldots x_{q}\right)+p_{20} l+p_{11} l f\left(x_{1}, x_{2} \ldots x_{q}\right) .
$$

Equation (21) can also be written as follows:

$$
f_{e r}\left(l, x_{1}, x_{2}, \ldots, x_{q}\right)=X\left[\Delta G, l, f\left(x_{1}, x_{2}, \ldots, x_{q}\right)\right]
$$

where $f_{e r}\left(l, x_{1}, x_{2} \ldots x_{q}\right)$ is the penalty function for the error data of the sampling point with armature displacement. The $i$ th MFL permeance or soft magnetic resistance can be defined as $q_{i}(x)$, where $x$ is the unknown quantity. Therefore, $w_{i}(x)$ can be obtained as follows:

$$
w_{i}(x)=f_{\text {eri }}(x)+q_{i}(x)
$$


where $w_{i}(x)$ is the improved DPM data, and it is very close to the FEM data.

\section{MFL Permeance Prejudge the Error Data Based on Kriging PIP}

To improve the overall calculation efficiency of the program, the predictive identification program is applied to prejudge the scatter data before employing the kriging base function.

The basis function can be defined as $f(x), a=\left(x_{1}, x_{2}, \ldots x_{n}\right) \in R^{n}$, where $f(x)$ is continuous and differentiable in the field of definition.

$$
\nabla f(x)(x-a)<0
$$

where $f(x)$ is the maximum with $x=x_{k}$.

Similarly,

$$
\nabla f(x)(x-a)>0
$$

where $f(x)$ is the minimum with $x=x_{k}$.

The extreme function can be analyzed according to Equations (24) and (25), and the gradient of $f(x)$ can be written as follows:

$$
\nabla f(x)=\left[\frac{\partial f(x)}{\partial x_{1}}, \frac{\partial f(x)}{\partial x_{2}}, \ldots, \frac{\partial f(x)}{\partial x_{n}}\right]^{T}
$$

Based on the characteristic of the first derivative, the adjacent points $x_{\mathrm{i}}$ and $x_{i+1}$ are the local maxima or minima. Therefore, the adjacent points are used as the criteria to judge the curve of the function. If the adjacent points of the function data are monotonically increasing, and the second derivative product is positive, this shows that the function is unimodal and it has a maximum. Therefore, it can be obtained as follows:

$$
\left\{\begin{array}{l}
\frac{\partial f(x)}{\partial x_{1}}=0, \frac{\partial f(x)}{\partial x_{2}}=0, \ldots, \frac{\partial f(x)}{\partial x_{n}}=0 \\
f\left(x_{i}\right)<f\left(x_{i+2}\right) \\
f\left(x_{i+1}\right)<f\left(x_{i+3}\right) \\
\frac{\partial^{2}(x) \partial^{2} f(x)}{\partial^{2} x_{i} \partial^{2} x_{i+2}}>0
\end{array},\right.
$$

Similarly,

$$
\left\{\begin{array}{l}
\frac{\partial f(x)}{\partial x_{1}}=0, \frac{\partial f(x)}{\partial x_{2}}=0, \ldots, \frac{\partial f(x)}{\partial x_{n}}=0 \\
f\left(x_{i}\right)>f\left(x_{i+2}\right) \\
f\left(x_{i+1}\right)>f\left(x_{i+3}\right) \\
\frac{\partial^{2}(x) \partial^{2} f(x)}{\partial^{2} x_{i} \partial^{2} x_{i+2}}>0
\end{array} .\right.
$$

From Equation (28), the function is unimodal and has a minimum.

Similarly,

$$
\left\{\begin{array}{l}
f\left(x_{i}\right)<f\left(x_{i+2}\right), f\left(x_{i}\right)>f\left(x_{i+2}\right) \\
\frac{\partial f(x)}{\partial x_{1}}=0, \frac{\partial f(x)}{\partial x_{2}}=0, \ldots, \frac{\partial f(x)}{\partial x_{n}}=0 \\
f\left(x_{i+1}\right)>f\left(x_{i+3}\right), f\left(x_{i+1}\right)<f\left(x_{i+3}\right)
\end{array} .\right.
$$

If the adjacent points of the function data are monotonically increasing or decreasing, then the function has a multi-crest. As such, the derivative can identify the extremum of the scatter data, and the shape of the multi-crest based on the number of extremum points.

To identify the more complicated scatter data curve, the standard function for numerical matching of the scatter data is applied. When the trend of the curve for the standard function is the same as the trend of the distribution for the scatter data, it can be said that the corresponding kriging basis function is practical for this type of standard function.

Based on the trend of the $\Delta G_{i}$ curve, the Schwefel function has similar characteristics. Figure 6 shows the standard Schwefel function. 


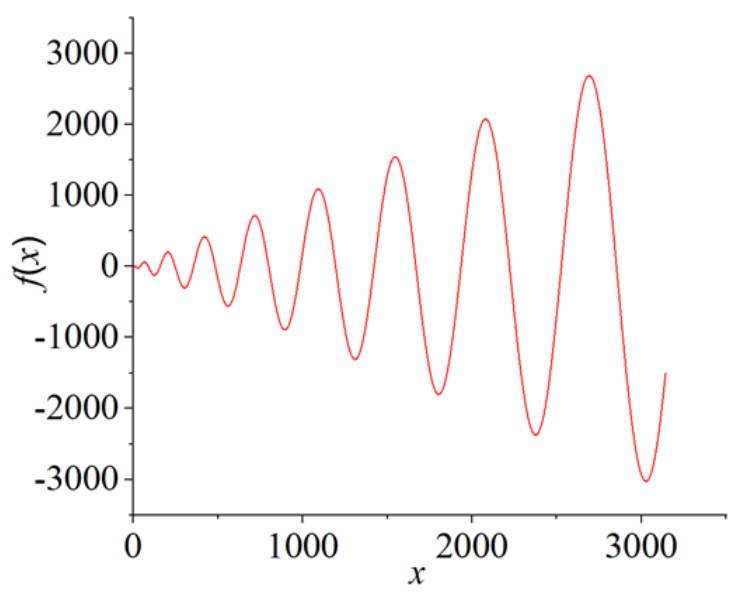

Figure 6. Standard Schwefel function.

The Schwefel function can be written as follows:

$$
f(x)=x \sin (\sqrt{|x|})
$$

From Equation (30), the parameters can be modified to have the following:

$$
f(x)=-1-2.6 x \sin (-3.8+15 \sqrt{|x-0.02|})
$$

The Schwefel functions are compared with $\Delta G_{3}$ and $\Delta G_{4}$, as shown in Figure 7.

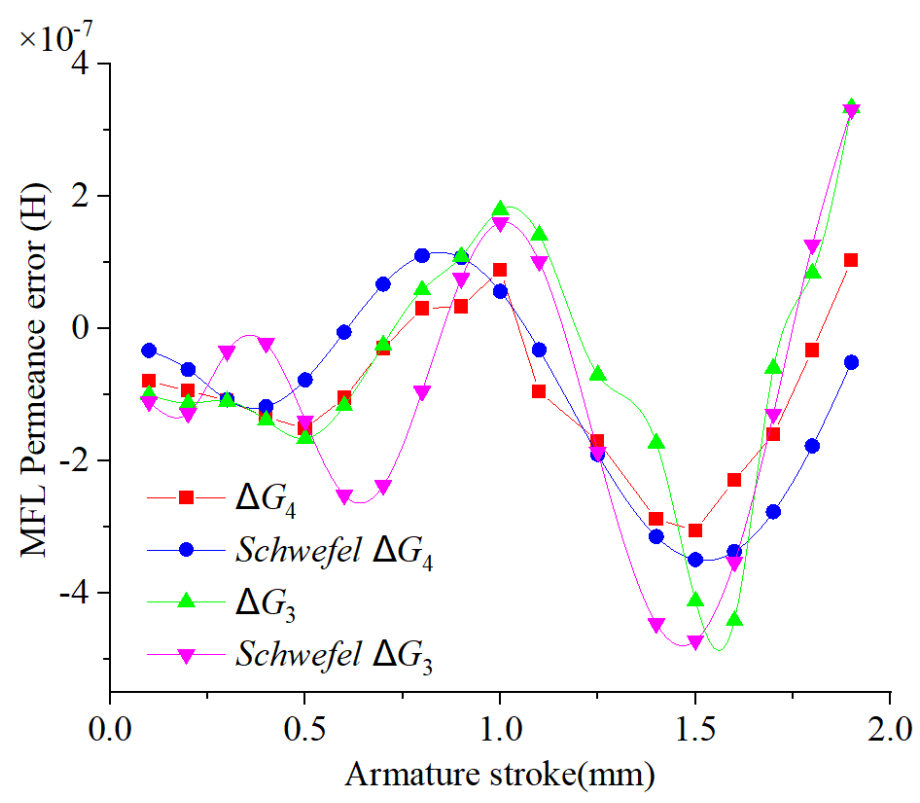

Figure 7. Schwefel function and $\Delta G_{3}, \Delta G_{4}$ curves.

The similarity between the Schwefel function, $\Delta G_{3}$ and $\Delta G_{4}$ can be obtained using the Tanimoto method. This method is stated as follows:

$$
T\left(x_{1}, x_{2}\right)=\frac{\sum x_{1 i} x_{2 i}}{\sum x_{1 i}^{2}+\sum x_{2 i}^{2}-\sum x_{1 i} x_{2 i}}
$$


By calculation, the similarity of $\Delta G_{3}$ with the Schwefel function is 0.7654 , and the similarity of $\Delta G_{4}$ with the Schwefel function is 0.7775 . The similarities of $\Delta G$ with the Schwefel function are further detailed in Table 2.

Table 2. Schwefel function and $\Delta G$ similarity.

\begin{tabular}{cccccccc}
\hline \multirow{2}{*}{ Schwefel Similarity } & $\Delta G_{1}$ & $\Delta G_{2}$ & $\Delta G_{3}$ & $\Delta G_{4}$ & $\Delta G_{5}$ & $\Delta G_{6}$ & $\Delta G_{7}$ \\
\cline { 2 - 8 } & 0.005 & 0.0662 & 0.7654 & 0.7775 & 0.1674 & 0.5698 & 0.0013 \\
\hline
\end{tabular}

In Table 2, the similarities of $\Delta G_{3}$ and $\Delta G_{4}$ with the Schwefel function are over 0.7, while the similarities of other $\Delta G_{1}, \Delta G_{2}, \Delta G_{5}, \Delta G_{6}$ and $\Delta G_{7}$ are the same as the trigonometric function. The similarities of $\Delta G$ are compared with the trigonometric function, as shown in Table 3.

Table 3. Trigonometric function and $\Delta G$ similarity.

\begin{tabular}{cccccccc}
\hline \multirow{2}{*}{ Trigonometric Similarity } & $\Delta G_{1}$ & $\Delta G_{2}$ & $\Delta G_{3}$ & $\Delta G_{4}$ & $\Delta G_{5}$ & $\Delta G_{6}$ & $\Delta G_{7}$ \\
\cline { 2 - 8 } & 0.8625 & 0.921 & 0.231 & 0.1922 & 0.8124 & 0.7958 & 0.7642 \\
\hline
\end{tabular}

It can be concluded that the similarities which are over 0.7 conform to the corresponding standard function. Hence, the kriging basis function can be selected. When the similarity of the $\Delta G$ curve and the Schwefel function is over $0.7, \Delta G$ can be modified by the Gaussian function. When the similarity of the $\Delta G$ curve and the trigonometric function is over $0.7, \Delta G$ can be modified by the Fourier function.

\section{The Error Correction of MFL Permeance and Soft Magnetic Resistance of Solenoid Valves}

To verify the practicality of the proposed method, the kriging model based on the basis function adjustment is used to modify the error of the MFL permeance and soft magnetic resistance data of the solenoid valve magnetic system. Substituting modified data into the DPM, the electromagnetic force can be obtained.

From Figure 8, it can be observed that the unmodified DPM permeance mean error reaches $13.1 \%$ (see Figure 2), and the modified DPM mean error reaches 4.7\%.

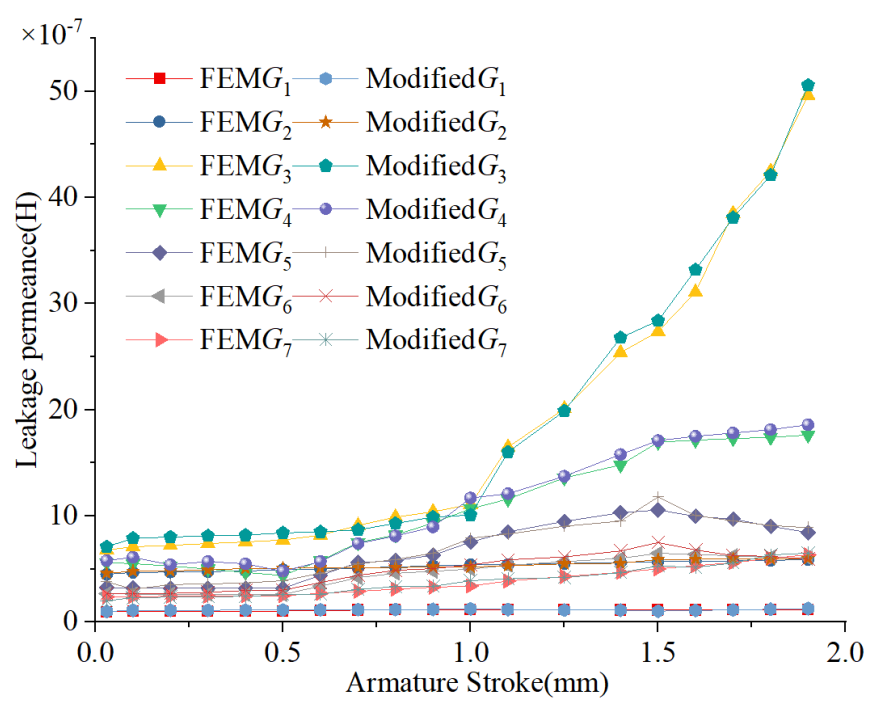

Figure 8. The leakage permeance of FEM and error modified distributed parameter model (DPM).

The soft magnetic resistance in $l a_{1}$ and $l a_{2}$ segments is taken as an example, without the presence of an excitation current in the coil and at different armature positions. Figure 9 
shows the soft magnetic resistance of FEM and the unmodified and modified DPM. The unmodified DPM permeance mean error reaches $9.94 \%$, while the modified DPM permeance mean error reaches $3.7 \%$.

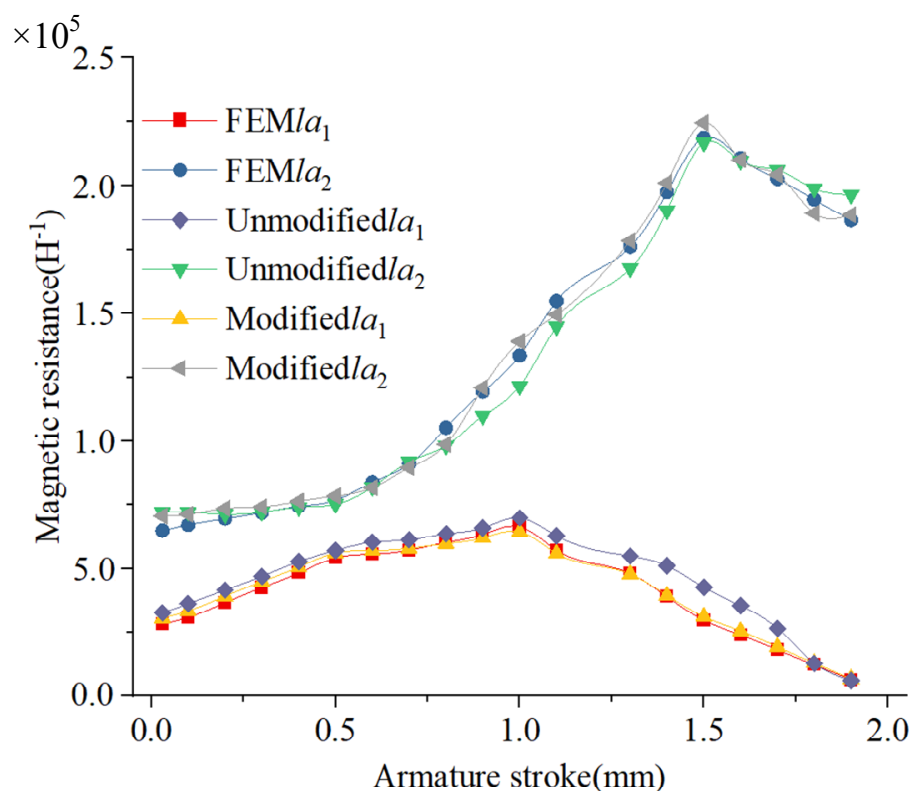

Figure 9. The soft magnetic resistance of FEM and unmodified and modified DPM.

The modified MFL permeance and soft magnetic resistance data are substituted into the DPM. The FEM results are compared with the modified and unmodified DPM results of the force on the armature for a coil excitation of $1800 \mathrm{AT}$ and $-1800 \mathrm{AT}$, as shown in Figure 10. The unmodified DPM mean error is $10.2 \%$, and the modified DPM mean error is $3.8 \%$. The FEM and measurement results mean error is $3 \%$.

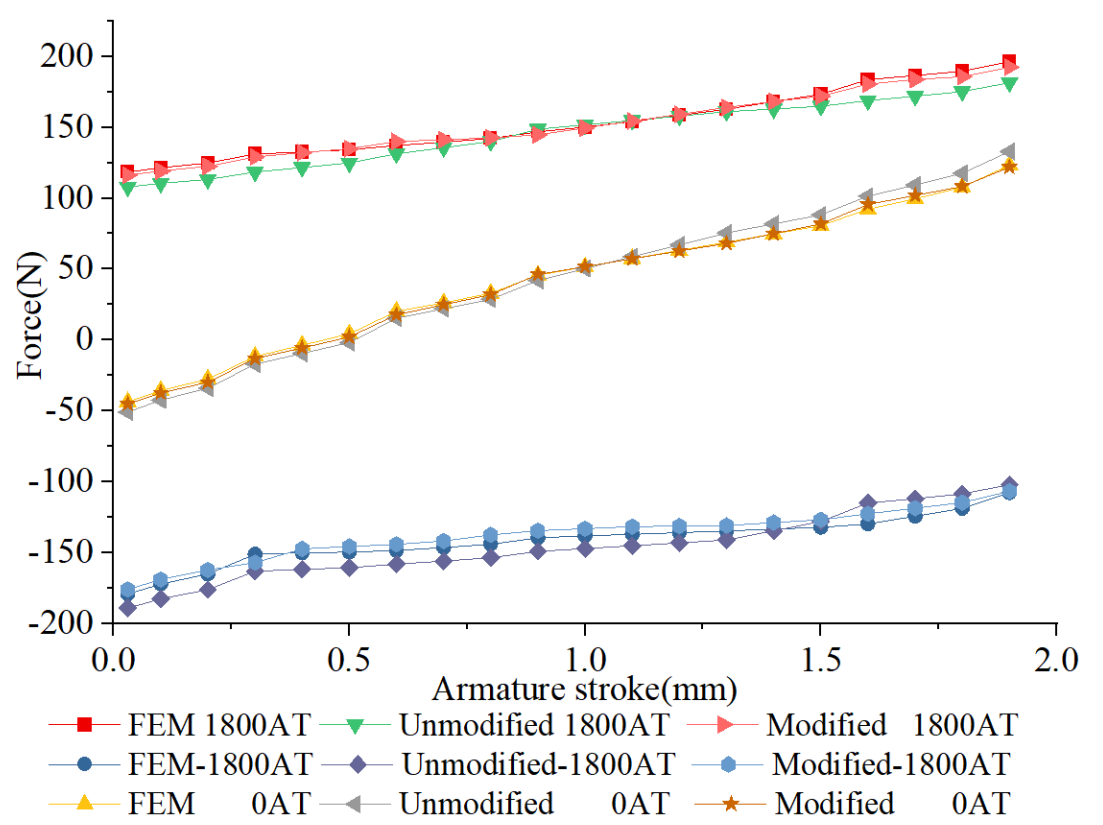

Figure 10. FEM and unmodified and modified DPM force data on the armature.

Figure 11 shows the flow chart of the improved method for the DPM of solenoid valves based on the kriging basis function PIP. An appropriate function is selected by the PIP for error correction, and by substituting modified data into the DPM, the electromagnetic force 
can be obtained. Under the same calculation conditions, the output force of one armature displacement point cost $420 \mathrm{~s}$ in the FEM, and $56 \mathrm{~s}$ in the DPM.

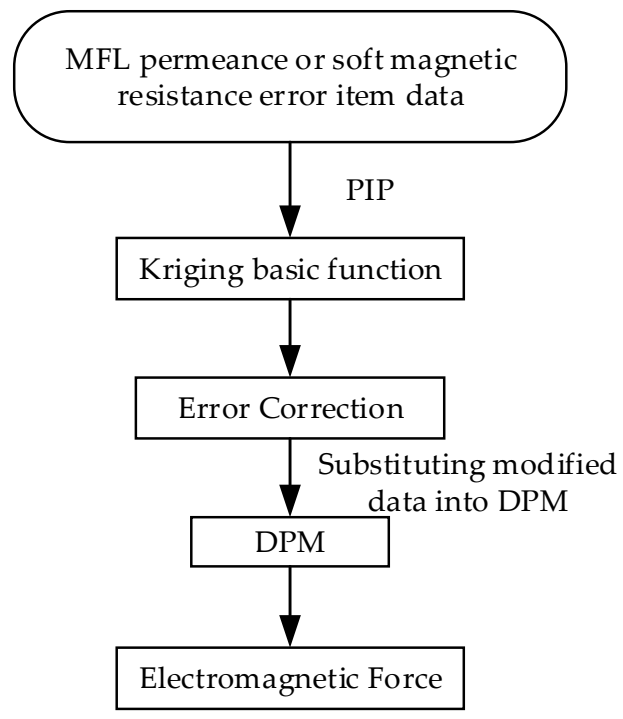

Figure 11. Flow chart of method for DPM based on kriging basis function predictive identification program (PIP) error correction.

\section{Conclusions}

To improve the calculation accuracy and efficiency of the solenoid valve DPM, an improved method for the DPM based on the kriging basis function PIP is proposed in this paper. From the experiments and the results, the following conclusions are drawn:

1. Based on the characteristics of the kriging basis function curve, the relationship between the kriging basis function and the MFL permeance error data can be obtained, and an appropriate function is selected by contrasting various basis functions with error data curves. Then it is applied to gain error compensation between the FEM and DMP data. The PIP is introduced to prejudge the error data by comparing the standard function to the selected basis function. The modified MFL permeance and the soft magnetic resistance data are then substituted into the DPM of the electromagnetic device to calculate the attraction force.

2. The proposed method can effectively improve the calculation accuracy of the solenoid valve electromagnetic system. Compared with the FEM data, the unmodified MFL permeance of the DPM mean error is $13.1 \%$, and the modified MFL permeance of the DPM mean error is $4.7 \%$. The unmodified MFL permeance of the DPM mean error is $9.94 \%$, and the modified MFL permeance of the DPM mean error is $3.7 \%$.

3. The results of the DPM solenoid valve electromagnetic system in the case study showed a significant improvement. Particularly, the calculation accuracy improved by reducing the DPM mean error from $10.2 \%$ to $3.8 \%$.

Author Contributions: Conceptualization, H.L., K.Z. and J.Y.; methodology, K.Z.; validation, X.F. and Y.R.; writing, K.Z.; supervision, H.L., K.Z. and J.Y.; funding acquisition, J.Y. All authors have read and agreed to the published version of the manuscript.

Funding: This research received no external funding.

Institutional Review Board Statement: Not applicable.

Informed Consent Statement: Not applicable.

Data Availability Statement: Please contact with the corresponding author.

Conflicts of Interest: The authors declare no conflict of interest. 


\section{References}

1. Hong, D.K.; Woo, B.C.; Koo, D.H.; Lee, K.C. Electromagnet Weight Reduction in a Magnetic Levitation System for Contactless Delivery Applications. Sensors 2010, 10, 6718-6729. [CrossRef] [PubMed]

2. Yang, X.M.; Guo, X.L.; Ouyang, H.J.; Li, D.S. A Kriging Model Based Finite Element Model Updating Method for Damage Detection. Appl. Sci. 2017, 7, 1039. [CrossRef]

3. Zhang, X.X.; Liu, G.D.; Wang, K.T.; Li, X.D. Application of a Hybrid Interpolation Method Based on Support Vector Machine in the Precipitation Spatial Interpolation of Basins. Water 2017, 9, 760. [CrossRef]

4. Zhang, D.W.; Li, W.L.; Wu, X.H.; Liu, T. An Efficient Regional Sensitivity Analysis Method Based on Failure Probability with Hybrid Uncertainty. Energies 2018, 11, 1684. [CrossRef]

5. Li, Y.K.; Song, B.W.; Mao, Z.Y.; Tian, W.L. Analysis and Optimization of the Electromagnetic Performance of a Novel Stator Modular Ring Drive Thruster Motor. Energies 2018, 11, 1598. [CrossRef]

6. Kwon, J.W.; Lee, J.H.; Zhao, W.L.; Kwon, B.I. Flux-Switching Permanent Magnet Machine with Phase-Group Concentrated-Coil Windings and Cogging Torque Reduction Technique. Energies 2018, 11, 2758. [CrossRef]

7. You, Y.M. Multi-Objective Optimal Design of Permanent Magnet Synchronous Motor for Electric Vehicle Based on Deep Learning. Appl. Sci. 2020, 10, 482. [CrossRef]

8. Pan, R.C.; Song, Z.Y.; Liu, B. Optimization Design and Analysis of Supersonic Tandem Rotor Blades. Energies 2020, 13, 3228. [CrossRef]

9. Bae, T.S.; Lee, K.H. Structural Design of a Main Starting Valve Based on the First Axiom. Int. J. Precis. Eng. Manuf. 2012, 13, 685-691. [CrossRef]

10. Lee, Y.M.; Choi, D.H. Design Optimization of an Automotive Vent Valve Using Kriging Models. Trans. KSAE 2011, 16, 1-9.

11. Kim, S.P.; Lee, K.H. Structural Optimization of a Manifold Valve for Pressure Vessel. J. Korean Soc. Precis. Eng. 2009, 26, 102-109.

12. Xue, X.F.; Wang, Y.Z.; Lu, C.; Zhang, Y.P. Sinking Velocity Impact-Analysis for the Carrier-Based Aircraft Using the Response Surface Method-Based Improved Kriging Algorithm. Adv. Mater. Sci. Eng. 2020, 6, 1-13. [CrossRef]

13. Rashki, M.; Azarkish, H.; Rostamian, M.; Bahrpeyma, A. Classification Correction of Polynomial Response Surface Methods for Accurate Reliability Estimation. Struct. Saf. 2019, 81, 101869. [CrossRef]

14. Sumiya, U.; Kwon, H.H. Development of bias correction scheme for high resolution precipitation forecast. J. Korea Water Resour. Assoc. 2018, 51, 575-584.

15. Wang, X.; Babovic, V.; Li, X. Application of Spatial-Temporal Error Correction in Updating Hydrodynamic Model. J. Hydro Environ. Res. 2017, 16, 45-57. [CrossRef]

16. Rathore, P.; Kumar, D.; Rajasegarar, S.; Palaniswami, M. Maximum Entropy-Based Auto Drift Correction Using High-and Low-Precision Sensors. ACM Trans. Sens. Netw. 2017, 13, 1-41. [CrossRef]

17. Alexeeff, S.E.; Carroll, R.J.; Coull, B. Spatial measurement error and correction by spatial SIMEX in linear regression models when using predicted air pollution exposures. Biostatistics 2016, 17, 377-389. [CrossRef] [PubMed]

18. Xia, B.; Lee, T.W.; Choi, K.; Koh, C.S. A Novel Adaptive Dynamic Taylor Kriging and Its Application to Optimal Design of Electromagnetic Devices. IEEE Trans. Magn. 2016, 52, 1-4. [CrossRef]

19. Zhang, C.; Qang, Z.; Shafieezadeh, A. Value of Information Analysis via Active Learning and Knowledge Sharing in ErrorControlled Adaptive Kriging. IEEE Access 2020, 8, 51021-51034. [CrossRef]

20. Chen, D.C.; Liu, D.Z.; Li, Y.H.; Meng, L.; Yang, X.J. Improve Spatiotemporal Kriging with Magnitude and Direction Information in Variogram Construction. Chin. J. Electron. 2016, 25, 527-532. [CrossRef]

21. Zhang, J.H.; Xiao, M.; Gao, L.; Zhang, Y. MEAK-MCS: Metamodel Error Measure Function based Active Learning Kriging with Monte Carlo Simulation for Reliability Analysis. In Proceedings of the 2019 IEEE 23rd International Conference on Computer Supported Cooperative Work in Design, Porto, Portugal, 6-8 May 2019.

22. Yan, Y.; Wang, J.; Zhang, Y.; Sun, Z. Kriging Model for Time-Dependent Reliability: Accuracy Measure and Efficient TimeDependent Reliability Analysis Method. IEEE Access 2020, 8, 172362-172378. [CrossRef]

23. Liu, X.; Li, X.S.; Huang, S.D. Parameters Optimization of the Permanent Magnet Linear Synchronous Machine Using Krigingbased Genetic Algorithm. In Proceedings of the 2019 22nd International Conference on Electrical Machines and Systems, Harbin, China, 11-14 August 2019.

24. Yin, J.; Ng, S.H.; Ming, K. A study on the effects of parameter estimation on kriging model's prediction error in stochastic simulations. In Proceedings of the 2009 Winter Simulation Conference, Austin, TX, USA, 13-16 December 2009.

25. Yu, J.C. Evolutionary algorithm using progressive Kriging model and dynamic reliable region for expensive optimization problems. In Proceedings of the 2016 IEEE International Conference on Systems, Man, and Cybernetics, Budapest, Hungary, 9-12 October 2016.

26. Pham, T.D. Kriging-based possibilistic entropy of biosignals. In Proceedings of the 20th European Signal Processing Conference, Bucharest, Romania, 27-31 August 2012.

27. Rivera, R. A Low Rank Gaussian Process Prediction Model for Very Large Datasets. In Proceedings of the 2015 IEEE First International Conference on Big Data Computing Service and Applications, Redwood City, CA, USA, 30 March-2 April 2015.

28. Jouhaud, J.C.; Sagaut, P.; Labeyrie, B. A kriging approach for CFD/wind-tunnel data comparison. J. Fluids Eng. Trans. ASME 2006, 128, 847-855. [CrossRef] 
29. Han, S.Q.; Song, W.P.; Han, Z.H. An improved WENO method based on Gauss-kriging reconstruction with an optimized hyper-parameter. J. Comput. Phys. 2020, 422, 109742. [CrossRef]

30. Cui, D.; Wang, G.Q.; Lu, Y.P.; Sun, K.K. Reliability design and optimization of the planetary gear by a GA based on the DEM and Kriging model. Reliab. Eng. Syst. Saf. 2020, 203, 107074. [CrossRef]

31. Shin, Y.J.; Lee, S.H.; Choi, C.H.; Kim, J.H. Shape Optimization to Minimize The Response Time of Direct-acting Solenoid Valve. J. Magn. 2015, 20, 193-200. [CrossRef]

32. Suh, Y.K. Multi-objective Optimization Strategy based on Kriging Metamodel and its Application to Design of Axial Piston Pumps. J. Adv. Mar. Eng. Technol. 2013, 37, 893-904.

33. Qin, W.J.; He, J.Q. Optimum design of local cam profile of a valve train. Proc. Inst. Mech. Eng. Part C J. Mech. Eng. Sci. 2010, 224, 2487-2492. [CrossRef]

34. Li, T.Z.; Yang, X.L. An efficient uniform design for Kriging-based Response Surface Method and its Application. Comput. Geotechics 2019, 109, 12-22. [CrossRef]

35. Trinchero, R.; Larbin, M.; Swaminathan, M.; Canavero, F.G. Statistical Analysis of the Efficiency of an Integrated Voltage Regulator by means of a Machine Learning Model Coupled with Kriging Regression. In Proceedings of the IEEE Workshop on Signal and Power Integrity, Chambery, France, 18-21 June 2019.

36. Liang, H.M.; Zhang, K.; You, J.X. Analytical Method for the Magnetic Field Line Distribution of a Fan-shaped Permanent Magnet and the Calculation of Leakage Permeance. J. Magn. 2017, 22, 395-405. [CrossRef]

37. You, J.X.; Zhang, K.; Zhu, Z.W.; Liang, H.M. Novel Design and Research for a High-retaining-force, Bi-directional, Electromagnetic Valve Actuator with Double-layer Permanent Magnets. J. Magn. 2016, 21, 65-71. [CrossRef]

38. Zhang, K.; Liang, H.M.; You, J.X.; Yu, H. Distributed Parameter Model for Electromagnetic Valve Actuator with Permanent Magnet. In Proceedings of the IEEE International Magnetics Conference, Dublin, Ireland, 24-28 April 2017.

39. Ye, X.R.; Chen, H.; Liang, H.M.; Chen, X.J.; You, J.X. Multi-Objective Optimization Design for Electromagnetic Devices with Permanent Magnet Based on Approximation Model and Distributed Cooperative Particle Swarm Optimization Algorithm. IEEE Trans. Magn. 2018, 54, 1-5.

40. Ye, X.R.; Chen, H.; Chen, C.; Zhai, G.F. Life-cycle Dynamic Robust Design Optimization for Batch Production of Permanent Magnet Actuator. IEEE Trans. Ind. Electron. 2020, 11, 3026294. [CrossRef] 